Indikation veröffentlicht hat. Diese wurden im Jahr 2005 erweitert und präzisiert.

Ein Aspekt wird bei der Diskussion um die Schlaf-Apnoe sehr oft vergessen: Die SBAS beeinträchtigt keineswegs nur die Gesundheit und Lebensqualität des Betreffenden, auch dessen Bettnachbar ist durch das starke Schnarchen erheblich beeinträchtigt. Die Problematik war bereits Gegenstand wissenschaftlicher Untersuchungen und es wurde zweifelsfrei dokumentiert, dass starkes Schnarchen beim Bettnachbarn eine erhebliche Fragmentierung des Schlafs bewirkt und damit die Schlafqualität beeinträchtigt.

\section{Den „Schlaf der anderen“ bedenken}

Die gestörte Nachtruhe kann dabei, ähnlich wie bei den Patienten selbst, infolge des Mangels an erholsamem Schlaf auch bei deren Lebenspartnern zu einer starken Müdigkeit führen mit entsprechenden Folgen wie Reizbarkeit, Nervosität und Konzentrationsstörungen. Diese wiederum können eine erhöhte Unfallneigung bedingen und bei entsprechender Disposition auch den Weg in manifeste Gesundheitsstörungen auf psychischer wie auch somatischer Ebene bahnen. Auch dieser Aspekt sollte Eingang in die Bewertung des Therapiever-

Herstellung und Anpassung von Unterkiefer-Protrusionsschienen

\title{
So findet man einen erfahrenen Zahnmediziner
}

Die Erstattung von Unterkiefer-Protrusionsschienen (UPS) erfolgt leider bislang noch nicht problemlos. Allerdings können die gesetzlichen Krankenkassen die Kosten für die Protrusionsschiene und deren Anpassung im Rahmen von Einzelfallentscheidungen übernehmen und tun dies inzwischen auch immer häufiger.

Die Schwerfälligkeit bei der Bezuschussung der Maßnahme und die nicht seltene Verweigerung einer vollständigen Kostenübernahme sind schwer nachvollziehbar. Denn die Schienen stellen bei entsprechender Indikationsstellung eine effektive und preisgünstige Behandlungsoption für Patienten mit leichter bis moderater Schlafapnoe dar und werden in aller Regel von den Betroffenen besser akzeptiert und mit besserer Compliance genutzt als die CPAP-Behandlung.
Problematisch dürfte aus Sicht der Krankenkassen vor allem sein, dass die Herstellung und Anpassung der UPS bislang nicht qualitätsgesichert erfolgt, so dass auch Zahnärzte und Kieferorthopäden ohne entsprechende Qualifikation und Erfahrung diese Therapieform anbieten können. Allerdings sind über die Webseite der Deutschen Gesellschaft Zahnärztliche Schlafmedizin (www.dgzs.de) qualifizierte und zertifizierte Behandler zu ermitteln, welche eine fundierte Ausbildung in diesem Spezialbereich der Zahnmedizin absolviert haben. Einzelne kleinere Betriebskrankenkassen haben die Vorteile - und wohl auch die Kostenvorteile - der UPS bei der Behandlung der Schlaf-Apnoe bereits erkannt und etablieren diese Therapieoption derzeit in ihrem Maßnahmenkatalog.

Dr. Elio Adler Zahnarzt, Berlin fahrens durch den Gemeinsamen Bundesausschuss (GBA) und in Überlegungen der Kostenübernahme durch die Krankenkassen finden.

Die effektive Behandlung des „Schnarchers" hat somit nicht nur für dessen eigene Gesundheit positive Konsequenzen, sondern ist förderlich auch für den Schlaf und damit letztlich für die Gesundheit - und wohl unbestreitbar für die Lebensqualität - seines Bettnachbarn.

\section{Bald mehr Geld für „,neue“ Patienten?}

Seit 15 Jahren setzt sich der Bundesverband der Pneumologen für ein transparentes, qualitätsbasiertes Vergütungsmodell in der gesetzlichen Krankenversicherung ein. Im neuen Versorgungsstrukturgesetz, das der Bundestag am 1. Dezember beschlossen hat, ist der Kern dieses Modells jetzt realisiert. Das zentrale Steuerungsmodell ist die Unterscheidung in bekannte "ALT"-Patienten und in mindestens drei Quartale nicht in der Praxis betreute "NEU“-Patienten.

Der Aufwand für die Diagnostik und Therapie von NEU-Patienten ist in aller Regel höher als für ALT-Patienten. Das soll in Zukunft besser vergütet werden können. Umgekehrt soll die Vergütung für regelmäßig in der Praxis behandelte bekannte Patienten sinken. Damit bildet das Hono- rarsystem in Zukunft den tatsächlichen Behandlungsbedarf besser ab und setzt Anreize, neue Patienten zu behandeln. Das Gesetz sieht auch vor, dass besondere Krankheitsverläufe durch differenziertere Pauschalen abgebildet werden. „Wir freuen uns über die Entscheidung des Gesetzgebers und sind gespannt auf die Umsetzung", erklärt dazu Dr. Andreas Hellmann, Vorsitzender des Bundesverbands der Pneumologen (BdP).

Die neue Regelung folgt nach seiner Darstellung im Kern dem Konzept für eine "Qualitätsgesicherte Einheitliche Liquidationsordnung für Lungenärzte", kurz QUELL, das der BdP vor 15 Jahren entworfen und seitdem in Zusammenarbeit mit der KBV und weiteren Berufsverbänden weiterentwickelt und immer wieder in die politische Diskussion eingebracht hat. Hellmann sieht in der ALT-NEU-Regelung auch einen direkten Nutzen für die Patienten: „Durch die realistischere Abbildung des Behandlungsbedarfs kann sich der Arzt noch ausführlicher um schwere Fälle kümmern. Die Routineversorgung von bekannten und gut eingestellten Patienten lässt sich gut im Team organisieren".

"Wir werden darauf achten, dass die Option „ALT/NEU“ nicht nur zur Honorarverschiebung auf dem Rücken der Vertragsärzte benutzt wird. Es muss sich für Patienten und Ärzte lohnen!" mahnt in dem Zusammenhang Dr. Michael Barczok aus Ulm, einer der Autoren von "QUELL". Weitere Infos unter www.pneumologenverband.de/quell.html 